Case Reports

Prashant Kaushal, MBBS

Department of Neurosurgery

Om Hospital and Research Centre

Chabahil, Kathmandu, Nepal

Rajiv Jha, MS, MCh

Department of Neurosurgery

Om Hospital and Research Centre

Chabahil, Kathmandu, Nepal

Pawan Kumar, MS

Department of Neurosurgery

Om Hospital and Research Centre

Chabahil, Kathmandu, Nepal

Gopal R Sharma, MS

Department of Neurosurgery

Om Hospital and Research Centre

Chabahil, Kathmandu, Nepal

Address for correspondence:

Prashant Kaushal, MBBS

Department of Neurosurgery,

Om Hospital and Research Centre

Chabahil, Kathmandu, Nepal

Email: pkaushal4u@gmail.com

Received, 8 January, 2017

Accepted, 19 January, 2017
Nepal Journal of Neuroscience 14:32-35, 2017

\section{Spontaneous Thoracic Spinal Epidural Hematoma: A Rare Cause of Paraparesis}

AcuteSpontaneous Spinalepiduralhematomas are rarely encountered in Neurosurgical practice. We report a case of seventy four year old man who presented to our emergency room with a four hour history of sudden onset severe back pain followed by progressive weakness of both lower limbs. On examination he had paraparesis of MRC (Medical Research Council) grade 4/5 with local tender at thoracic spine. Magnetic Resonance Imaging (MRI) of thoracic spine revealed posterior epidural mass compressing the cord from D8 to D10 level suggestive of epidural hematoma. He underwent decompressive laminectomy and excision of epidural mass which turned out to be hematoma on histopathology. There was immediate improvement in neurological function after surgery. Thus the diagnosis of spinal epidural hematoma must be kept in mind in case of sudden back pain with symptoms of spinal cord compression. Early recognition, accurate diagnosis and prompt surgical treatment may result in significant improvement of neurological functions.

Key Words: laminectomy, Magnetic Resonance Imaging, paraparesis, spontaneous spinal epidural hematoma, thoracic spine

malformation or tumor, ${ }^{7,11}$ however in up to $40 \%$ of cases, no underlying cause can be identified. ${ }^{3}$ Although cases of successful non operative treatment have been reported, prompt surgical evacuation of epidural clot remains the gold standard treatment. ${ }^{4}$

\section{Case Report}

A 74-year-old man presented to our emergency room with a four hours history of sudden onset severe backache followed by gradually progressive weakness and paraesthesia with tingling sensation of both lower 


\section{Kafle et al}

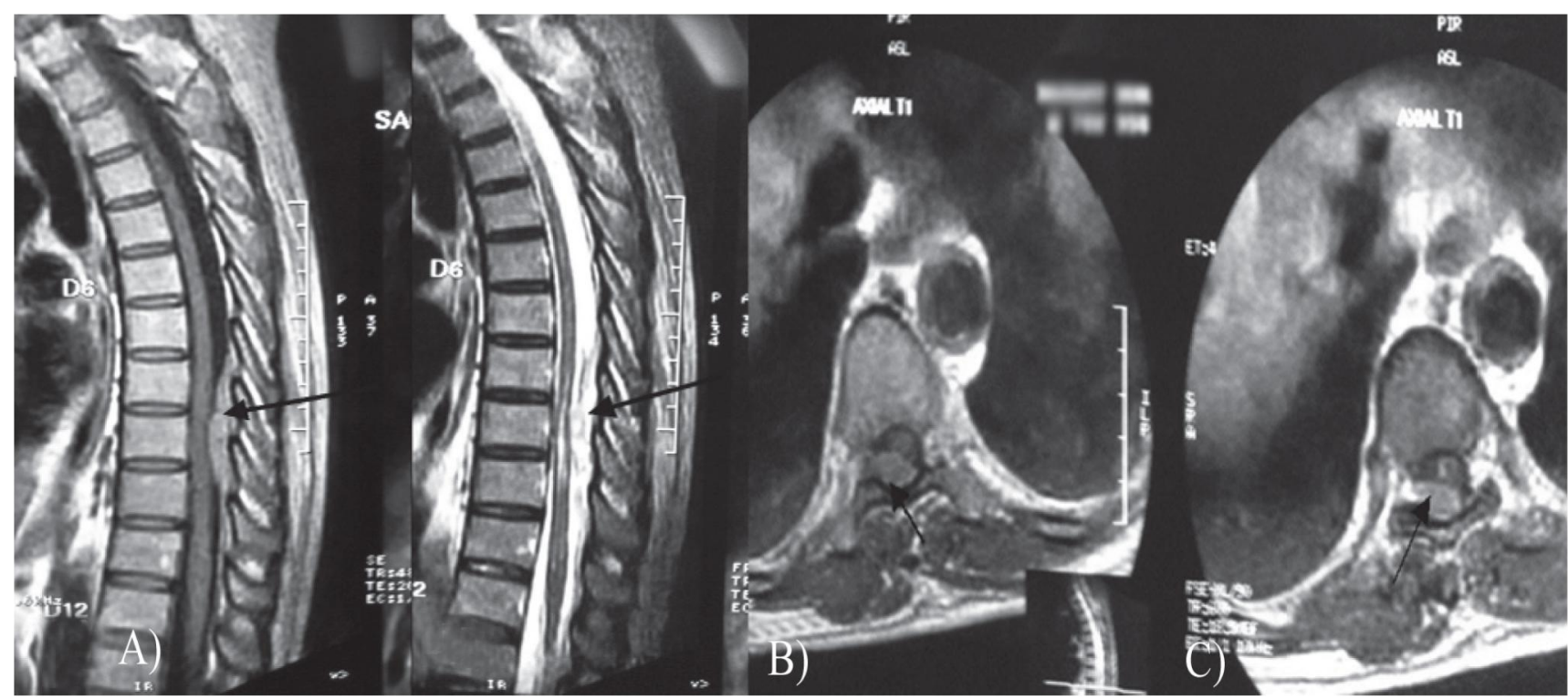

Figure 1: A,B) Preoperative sagittal and C) axial MRI images showing epidural hematoma from D8 to DI0 level

limbs. There was no recent history of trauma or use of oral anticoagulant. He had no history of hypertension, bleeding dyscrasia or spinal surgery in the past. On general examination, the patient was found to be fit with no chest, cardiovascular or abdominal abnormalities; however tenderness was noted in the mid thoracic segment of spine. The neurological examination revealed normal higher mental functions, cranial nerves and upper extremities however he had paraparesis of MRC (Medical Research Council) grade $4 / 5$ with bilateral positive Babinski's signs. There was no bowel and bladder incontinence

Theroutinehematological, biochemicaland coagulation profile were unremarkable. Magnetic Resonance Imaging (MRI) of thoracic spine demonstrated an abnormal soft tissue mass in the posterior epidural space compressing the cord and extending from D8 to D10 level measuring about $63 \times 12.4 \times 11.6 \mathrm{~mm}$ which was slightly hyperintense to cord in $\mathrm{T} 1$ and heterogeneously hyperintense in $\mathrm{T} 2$ and STIR with minimal contrast enhancement (Figure 1). Based on clinical presentation, hematological investigation and imaging findings, a provisional diagnosis of spontaneous spinal epidural hematoma of thoracic spine was suspected. The patient underwent decompressive laminectomy from D8 to D10 with total excision of a dark extradural mass compressing the spinal cord (Figure 2) that proved histopathologically to be an organized hematoma without any evidence of a tumor or vascular abnormality (Figure 3). Post-operatively the patient made an uneventful recovery and was discharged without any neurological deficit ten days after surgery. At a follow up of six months, the patient was asymptomatic without any neurological deficits.

\section{Discussion}

Spontaneous Spinal epidural hematoma is one of the rarest of all kinds of bleeding occurring within the craniospinal cavity. ${ }^{3}$ Jackson was the first to describe the term spinal epidural hematoma for the first time in $1869 .{ }^{8}$ Since then many spontaneous epidural hematoma cases have been reported in literature. Holtas et al. ${ }^{7}$ reported the incidence of 0.1 patients per 100,000 populations per year and less than $1 \%$ of spinal epidural space occupying lesion. Although patients of all ages may be affected, one half of cases occur between the ages of 50 and 80 years and hematoma is slightly predominant in men with common locations being cervicothoracic and thoracolumbar junctions. ${ }^{5}$ Our present case had SSEH in the thoracic region in a 74 year male patient.

The true pathogenesis of SSEH is unclear. The predisposing factors responsible for SSEH include coagulopathy, hypertension, increased venous pressure (such as during coughing, sneezing, lifting or vomiting) anticoagulant therapy, vascular malformation or tumor ${ }^{7}$ $11,4,5$ however; in up to $40 \%$ of cases, no underlying cause can be identified. ${ }^{3}$ Groen et al reviewed 199 cases of nontraumatic spinal epidural hematoma and they couldn't find any cause in 103 patients. ${ }^{5}$ Our present case is also thought to be of unknown etiology as none of the above predisposing factors were noted during the etiological workup.

The exact origin of SSEH is still controversial while the most popular hypothesis postulates that spinal epidural venous plexus is the source of this type of hematoma ${ }^{5}$ because the hematoma remains limited to a 


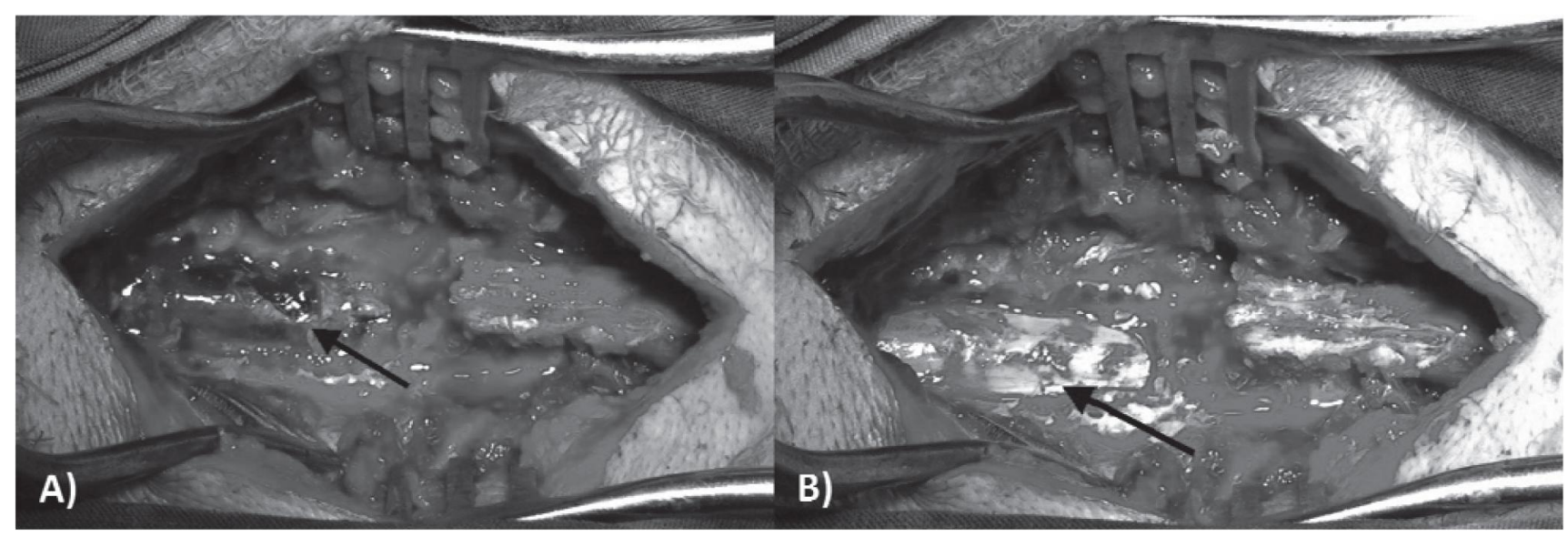

Figure 2: A) Intraoperative images after laminectomy showing the epidural clot compressing the cord, B) spinal cord after removal of clot

small number of segments i.e. three to four and is most frequently situated posterolaterally, and epidural veins are valveless and consequently not protected against variations of abdominal or thoracic pressure. Groen et al. ${ }^{5}$ described that posterior internal vertebral venous plexus played an important role in the etiology of SSEH. The posterior plexus is much bigger than the anterior internal spinal venous plexus and the anterior plexus is covered by posterior longitudinal ligament and to some extent it is sheltered from epidural space. This fact also supports the theory of Groen et al. However; Beatty and Winston, ${ }^{1}$ on the other hand, suggested that an arterial source of bleeding originating from the extensive network of epidural arteries better explains the precipitous neurological deterioration seen clinically. They argued that the pressure in the epidural cervical veins is lower than intrathecally, and thus a venous bleeding cannot compress the cervical spinal cord. Proof of either theory has been difficult to obtain because of a lack of angiographic documentation of the lesion as well as inconsistent explanation of the cause of the surgically removed clot.

The first clinical symptom of acute spinal epidural hematoma is the sudden onset of severe back and/or neck pain often with radicular component. Motor and/or sensory deficit follow, usually progressing over the course of several hours. In some cases, bowel and bladder may also be involved. ${ }^{14}$ In our case, the neurological deficits were observed at 4 hours after onset of symptoms; however there was no bowel and bladder involvement.

Despite the clinical syndrome of SSEH is relatively characteristic, its exact diagnosis may be difficult. Myelography, spinal CT and CT Myclography allow diagnosis of an extradural spinal cord compression, but it can't differentiate other causes. MRI is the diagnostic method of choice because of its sensitivity in depicting soft tissue lesion and its ability to survey and cover the full extent of hematoma ${ }^{2}$. Although idiopathic cases accounts for approximate $40 \%$ of all SSEH, MRI and angiography should be performed to confirm the cause. Liu et al. reported only one patient of $23 \mathrm{SSEH}$ patients had coagulopathy and six patients of 23 had vascular malformation. ${ }^{10}$ In addition, coagulation profile must be performed to rule out coagulopathy. Our patient had neither coagulation abnormality nor hematologic disease. He was not under anticoagulants and had no history of drug abuse. However we were unable to perform angiography due to limited time.

The therapy of choice for SSEH has been prompt surgical evacuation of hematoma by laminectomies; usually with favorable results. The indications for surgical management are progressive deterioration of neurologic functions and mass effect on MRI images. Favorable factors for SSEH are incomplete neurological injury at the time of preoperative status and a short operative time interval. ${ }^{12}$ Groen and Van Alphen ${ }^{4}$ reviewed 333 cases of SSEH and concluded that the interval of Pre-operation was one of the key factors to predict the prognosis. They indicated that in cases with incomplete neurological deficits, surgery should be performed no more than 48 hours after initial symptom onset; if initial neurological deficit were complete, surgery should shift to earlier than 36 hours. Similarly, Lawton et al. ${ }^{9}$ studied the relationship between surgical timing and neurological outcome, and concluded that the overall surgical outcome was inversely proportional to the time interval from the symptom onset to surgery and the duration of maximum deficit, which both reflected the duration of spinal cord compression. They argued that the interval should be bought forward to 12 hours.

In recent publications, some cases of successful conservative treatment of SSEH have been reported. ${ }^{13,6}$ This approach may be suitable in patients with only slight neurologic symptoms and a relatively small hematoma size. Pahapill and Lownie ${ }^{13}$ have reported a case with 


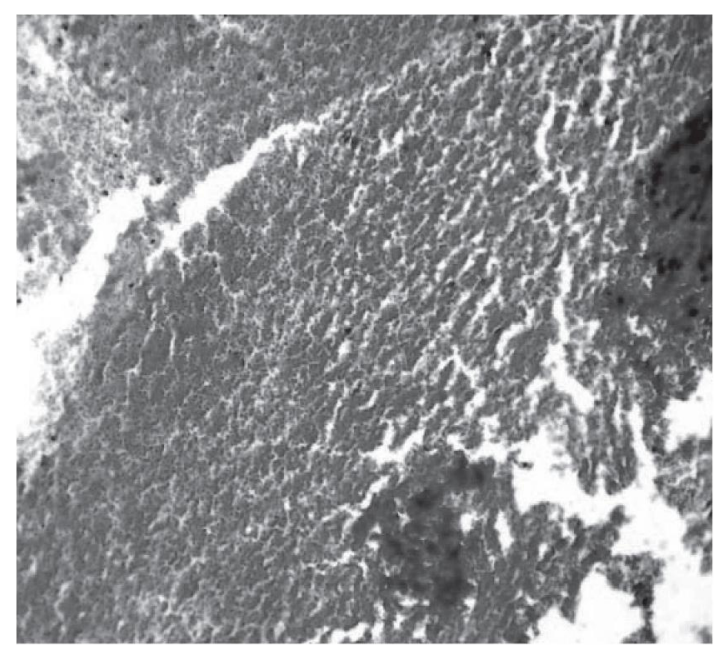

Figure 3: Histological picture of the mass showing organized blood clots.

significant neurologic deficit that resolved within 24 hour, although a markedly sized thoracic hematoma was present. They excluded a vascular malformation by angiography and followed a conservative regimen. The patient recovered fully and the hematoma resolved completely. They concluded that conservative treatment may play a role in patients who show a significant and sustained reversal of their symptoms in early phase of disease. ${ }^{13}$ Similarly Groen et $\mathrm{al}^{6}$ reviewed 64 case of conservatively treated SSEH and concluded that $84 \%$ experienced a complete recovery. However the mainstay of treatment remains surgery. The decision for conservative therapy is based on the severity of neurological deficits and on the clinical course. ${ }^{6}$ In our present case, we did not choose the conservative therapy due to rapidly increasing neurological deficit and significant spinal cord compression in MRI.

\section{Conclusions}

SSEH is a rare but disabling entity and should be suspected in patients with sudden onset severe back or neck pain followed by neurological deficits. The primary intervention is surgery unless the neurological status is retrieved in a short period.

\section{References}

1. Beatty RM, Winston KR. Spontaneous cervical epidural hematoma. A consideration of etiology. $\mathbf{J}$
Neurosurg 61:143-148, 1984

2. Boukobza M, Guichard JP, Boissonet M et al. Spinal Epidural hematoma: Report of 11 cases and review of literature. Neuroradiology 36: 456-459, 1994

3. Foo DK, Rossier AB. Preoperative neurological status in predicting surgical outcome of spinal epidural hematomas. Surg Neurol 15:389-401, 1981

4. Groen RJM, van Alphen HAM. Operative treatment of spontaneous spinal epidural haematomas: a study of factors determining postop-erative outcome. Neurosurgery 39: 494-509, 1996

5. Groen RJ, Ponssen H. The spontaneous spinal epidural hematoma: A study of the etiology. J Neurol Sci 98:121-138, 1990

6. Groen RJM. Non-operative treatment of spontaneous spinal epidural hematomas: a review of the literature and a comparison with operative cases. Acta Neurochir 146:103-110, 2004

7. Holtas S, Heiling M, Lönntoft M. Spontaneous spinal epidural hematoma: findings at MR imaging and clinical correlation. Radiology 199:409-413, 1996

8. Jackson R. Case of spinal apoplexy. Lancet 2:5-6, 1869

9. Lawton MT, Porter RW, Heiserman JE et al. Surgical management of spinal epidural hematoma: relationship between surgical timing and neurological outcome. J Neurosurg 83:1-7, 1995

10. Liu Z, Jiao Q, Xu J, Wang X, Li S, You C. Spontaneous spinal epidural hematoma: Analysis of 23 cases. Surg Neurol 69:253-260, 2008

11. Miyagi Y, Miyazono M, Kamikaseda K. Spinal epidural vascular malformation presenting in association with a spontancously resolved acute epidural hematoma. J Neurosurg 88:909-911, 1998

12. Nourbakhsh A, Chaljub G, Garges KJ. Spontaneous cer-vical epidural hematoma masquerading as an abscess on mag-netic resonance imaging scan. J Manipulative Physiol Ther 32:391-395, 2009

13. Pahapill PA, Lownie SP. Conservative treatment of acute spontaneous spinal epidural hematoma. Can J Neurol Sci 25:159 -163, 1998

14. Takahashi K, Koiwa F, Hironori $T$ et al. A case of spontaneous epidural hematoma in chronic renal failure patient undergoing hemodialysis: successful outcome with surgical management. Nephrol Dial Transplant14: 2499-2501, 1999 